\title{
Avaliação do vocabulário receptivo de crianças pré-escolares
}

\author{
An evaluation of receptive vocabulary \\ in preschool children
}

\author{
Maria Vanderléia Matos ARAÚJO' \\ Márcia Regina Fumagalli MARTELETO² \\ Teresa Helena SCHOEN-FERREIRA ${ }^{3}$
}

\begin{abstract}
Resumo
Linguagem receptiva é a capacidade de o indivíduo compreender o que ouve e lê. Este artigo objetiva avaliar o desempenho de crianças pré-escolares quanto ao vocabulário receptivo. Participaram 159 alunos - 56\% do $1^{\circ}$ estágio e 44\% do $3^{\circ}$ estágio - de uma escola de Educação Infantil do Município de São Paulo. Foi utilizado o Teste de Vocabulário por Imagens Peabody, que avalia o desenvolvimento lexical no domínio receptivo. Após autorização da direção da escola e dos pais, as crianças foram avaliadas individualmente. Houve um desempenho inferior ao esperado para a idade em $61 \%$ das crianças. Os alunos do $1^{\circ}$ estágio saíram-se melhor do que os do $3^{\circ}$ estágio. O gênero da criança e a escolaridade da mãe não interferiram no desempenho do teste. Conclui-se que é importante, já na pré-escola, um trabalho mais intensificado com vocabulário para as crianças desenvolverem seu potencial comunicativo.
\end{abstract}

Unitermos: Educação infantil. Linguagem infantil. Vocabulário.

\begin{abstract}
Receptive language is the individual's capacity to understand what he hears or reads. Objective: to evaluate the performance of kindergarten children in terms of their receptive vocabulary. 159 students from a public school participated in the study and responded to the Peabody Test that evaluates the lexical development in the receptive domain. Procedure: after obtaining authorization from the school principal's office and parents, the children were evaluated individually. Results: $61 \%$ of the children showed a lower performance than expected for their age. The $1^{\text {st }}$ grade students demonstrated better results than those in the $3^{\text {rd }}$ grade. The child's gender and the mothers'level of education did not interfere in the test performance. Conclusion: as early as kindergarten, it is already important to work on vocabulary, in order to develop the children's potential for communication.
\end{abstract}

Uniterms: Child reading. Child language. Vocabulary.

A linguagem é definida como um sistema arbitrário de símbolos que serve para expressar ideias e sentimentos e mediar o comportamento (Luque \& Vila,
2004). Deve ser concebida no contexto da interação social não simplesmente como meio de transmissão de informação, mas como projeção das próprias pessoas, veículo

\section{$\boldsymbol{\nabla \nabla \nabla \nabla}$}

1 Universidade Federal de São Paulo, Departamento de Fonoaudiologia, São Paulo, SP, Brasil.

2 Universidade Nove de Julho, Curso de Psicologia, Departamento de Saúde. São Paulo, SP, Brasil.

3 Universidade Federal de São Paulo, Departamento de Pediatria, Centro de Atendimento e Apoio ao Adolescente. R. Joaquim Távora, 550, ap. 84, São Paulo, SP, Brasil. Correspondência para/Correspondence to: T.H. SCHOEN-FEREIRA. E-mail: <teresaschoen.dped@epm.br>. 
de trocas, de relações, como meio de representação e comunicação, observando seus significados intrínsecos. Nesse sentido, a linguagem possui uma dinâmica que contribui para o desenvolvimento cognitivo infantil, podendo ser estudada sob pontos de vista distintos (Fitch, 2007; Schirmer, Fontoura \& Nunes, 2004). A teoria do processamento de informação investiga as diferenças individuais observáveis no desempenho de diversas tarefas, estudando separadamente habilidades cognitivas, incluindo a linguagem (Sternberg \& Grigorenko, 2006).

Como um sistema finito de princípios e regras, a linguagem permite que um falante codifique significado em sons e que um ouvinte decodifique sons em significados (Bee, 2003, Schirmer et al., 2004). A compreensão deve ser vista como um processo que exige que a criança infira os significados a partir de contextos plausíveis (Johnson et al., 2009; Sternberg \& Grigorenko, 2004).

A linguagem receptiva diz respeito à capacidade de a criança compreender a linguagem, em contraste com sua capacidade de expressá-la, denominada linguagem expressiva: habilidade da criança falar e se comunicar oralmente (Bee, 2003, Feldman, Campbell, Kus-Lasky \& Rockette, 2005, Macedo, Capovilla, Duduchi, D'Antino \& Firmo, 2006, Smeekens, Riksen-Walraven \& Bakel, 2008). A linguagem receptiva compreende o entendimento da entonação, da melodia da voz do outro durante a fala e do significado das palavras em seus diferentes contextos.

Muito antes de começar a falar, a criança está habilitada a usar o olhar, a expressão facial e o gesto para comunicar-se com os outros. Aos poucos, desenvolve a capacidade para discriminar os sons da fala. A aprendizagem do código linguístico baseia-se no conhecimento que a criança vai adquirindo do mundo ao seu redor, sendo resultante da interação bastante complexa entre capacidades biológicas, inatas e estimulação ambiental (Feldman et al., 2005; Nogueira, Fernández, Porfírio \& Borges, 2000; Olds et al., 2004).

Os modelos de aquisição de linguagem infantil preveem interação entre níveis linguísticos, fonológicos, vocabulares, sintáticos e semânticos. As dimensões de organização adequadas para as primeiras fases de compreensão e produção linguísticas devem ser encontradas nas representações memorizadas da criança para

170 eventos ou experiências específicos (Giusti \& Befi-Lopes,
2008, Smeekens et al., 2008). Como acreditam Mota e Castro (2007), o processo de escolarização provoca mudanças no pensamento. A linguagem receptiva é necessária para a compreensão das instruções dadas pelo professor, sejam elas curtas, compridas, simples ou complexas. O ingresso em uma escola de educação infantil aumenta as exigências de clareza e compreensão das produções verbais, especialmente o vocabulário receptivo (Hage, Joaquim, Carvalho, Padovani \& Guerreiro, 2004; Luque \& Vila, 2004).

Espera-se que, no período pré-escolar, as crianças desenvolvam produções linguísticas mais claras e compreensivas, aumentem seu vocabulário, usem melhor as flexões gramaticais e comecem a se preocupar com uma sintaxe mais complexa. Antes de ingressarem no ensino fundamental, já devem ter tido convívio com um repertório linguístico variado, acesso à linguagem escrita e já deve haver um domínio completo do repertório fonético (Luque \& Vila, 2004). As crianças parecem ter muitos conceitos sobre o mundo antes de terem palavras para eles, mas aprender novas palavras também cria novos conceitos (Rhoades, Greenberg \& Domitrovich, 2009).

O modo como os pais e professores falam com a criança, a quantidade e qualidade do vocabulário utilizado e a experiência com a linguagem e o ambiente possibilitam a aquisição de palavras novas. Pode-se dizer que a compreensão da linguagem por parte das crianças acontece bem antes da produção expressiva oral ou escrita (Berk, 2001; Li, Zhao \& Whitnney, 2007; Romski \& Sevcik, 2005). Inclusive há muitas palavras que podem ser compreendidas embora jamais usadas. As crianças parecem utilizar outros componentes da linguagem para compreender o significado das palavras, como a gramática, escutando palavras e deduzindo o significado a partir de como elas são utilizadas na frase. 0 número de palavras compreendidas é o dobro do de palavras emitidas, comprovando que o desenvolvimento da compreensão é mais rápido que o da emissão (Benedict, 1979).

Relata-se que as crianças pequenas aprendem uma média de nove palavras por dia, atingindo 14 mil palavras no seu vocabulário de compreensão pela idade de 6 anos (Berk, 2001; Chapman, 1996). As crianças diferem no ritmo do desenvolvimento do vocabulário e da gramática, diferenças explicadas pela hereditariedade e 
pelas influências ambientais. Apesar dessas variações do desenvolvimento inicial, a maioria das crianças já fala bem aos cinco ou seis anos de idade (Bee, 2003). Muitos estudos mostram que as meninas estão um pouco à frente dos meninos em relação à linguagem até os dois anos de idade. Depois, gradualmente, as diferenças vão desaparecendo (Berk, 2001).

A boa capacidade verbal está positivamente correlacionada à aquisição de conhecimentos e também prediz o sucesso fora da área acadêmica (Atance \& Jackson, 2009; Rhoades et al., 2009; Romski \& Rose, 2005; Sternberg \& Grigorenko, 2006). As pessoas com capacidade verbal geral superior são mais capazes de captar o significado das palavras a partir de indícios. Para descobrir o significado de uma palavra desconhecida, uma pessoa deve ser capaz de reagir ao contexto no qual a palavra aparece (Sternberg \& Grigorenko, 2004, 2006). Belintante (2000) afirma que, para o indivíduo transitar entre os diferentes ambientes que frequenta, necessita de um vocabulário mais amplo.

A ida da criança pequena para a escola propicia benefícios, pois as situações escolares podem ser ricas em experiências sociais e de aprendizagem. A possibilidade de vivenciar situações diversificadas e sistemáticas de comunicação tende a acelerar a aquisição da linguagem. Por meio da linguagem, a criança se torna capaz de compreender o contexto cultural e nele se inserir. As relações e normas culturais circulam na linguagem, que ocupa, portanto, um papel central na socialização do indivíduo (Hage et al., 2004; Johnson et al., 2009; Olds et al., 2004; Rhoades et al., 2009).

O crescimento social da criança implica sua inserção e participação em grupos que proveem situações de interação que estruturam seu ambiente e criam para ela necessidades cada vez mais avançadas de comunicação (Johnson et al., 2009; Olds et al., 2004; Rhoades et al., 2009; Sternberg \& Grigorenko, 2004). As primeiras palavras compreendidas pela criança estão relacionadas a pessoas, objetos, eventos, cenários e afeto. Atrasos na compreensão desses conceitos limitarão a compreensão, aquisição e uso de linguagem (Chapman, 1996).

$O$ atraso de linguagem pode ser identificado a partir dos dois anos de idade por meio da avaliação do número de palavras faladas e/ou compreendidas (F.C. Capovilla \& A.S.G. Capovilla, 1997) e pode envolver problemas na incorporação de novas palavras ao vocabulário e, se não tratado, pode ter consequências cognitivas, sociais e emocionais graves (Johnson et al., 2009; Olds et al., 2004; Rhoades et al., 2009). As alterações da linguagem situam-se entre os mais frequentes problemas do desenvolvimento, atingindo 19\% das crianças pré-escolares e 16\% das escolares, segundo Perissinoto, Schoen-Ferreira e Marteleto (2007).

A manifestação do retardo de linguagem ocorre na forma de uma evolução não satisfatória ou dificultosa, podendo atingir o aspecto de compreensão e expressão verbal: vocabulário restrito, dificuldades em elaborar frase, uso pouco frequente da linguagem, dificuldades de compreensão, inabilidade para relatar fatos ou acontecimentos vivenciados, narrativa truncada e apoiada em gestos, fala ininteligível, geralmente acompanhada de distúrbios articulatórios (Johnson et al., 2009, Schirmer et al., 2004).

Duncan, Brooks-Gunn e Klebanov (1994) estudaram o desenvolvimento cognitivo de crianças aos cinco anos de idade, levando em conta a baixa renda familiar, a duração das privações e a escolaridade materna, que, quando de melhor nível, exerceu efeito benéfico, enquanto a baixa renda e a duração das privações, efeito negativo significativo. Cardoso, Pedromônico, Silva e Puccini (2003) constataram que a escolaridade materna interfere positivamente sobre o desenvolvimento da linguagem da criança pré-escolar.

Um estudo realizado em creches e pré-escolas no Embu, uma cidade-dormitório da Grande São Paulo, mostrou que 44,3\% das crianças apresentaram linguagem receptiva abaixo da média esperada para a idade. Como fatores associados ao melhor desempenho, observaram-se a idade da criança e a escolaridade materna (Basílio, Puccini, Silva \& Pedromônico, 2005).

As crianças menos expostas à linguagem ou expostas a uma linguagem menos variada em seus primeiros anos de vida não parecem alcançar, posteriormente, as outras em vocabulário (Bee, 2003; Berk, 2001). Dias, Enumo e Turini (2006) observaram que as crianças de 5 a série não haviam adquirido, nas séries anteriores, as habilidades necessárias para cursarem com sucesso a série em que se encontravam. Fracasso escolar em séries mais avançadas muito possivelmente seja decorrente de falhas no processo inicial de aquisição das 
habilidades comunicativas necessárias à boa aprendizagem.

O DSM IVTR (American Psychiatric Association, 2002) identifica o transtorno da linguagem receptiva, situação em que a compreensão da linguagem pela criança está demonstrada por escores acentuadamente abaixo das medições padronizadas, individualmente administradas. As características linguísticas do transtorno variam de acordo com a gravidade e a idade da criança e incluem uma fala de quantidade limitada, vocabulário restrito, dificuldade em adquirir novas palavras, erros na busca da palavra correta ou de vocabulário, frases abreviadas, estruturas gramaticais simplificadas, variedades limitadas de estruturas gramaticais e/ou tipos de frases, omissões de partes críticas das frases, uso de uma ordem inusitada das palavras e desenvolvimento lento da linguagem. As crianças com essa espécie de prejuízo frequentemente começam a falar tarde e atravessam mais lentamente do que o habitual os vários estágios do desenvolvimento da linguagem expressiva. O tipo evolutivo de transtorno da linguagem receptiva geralmente é identificado por volta dos três anos de idade.

O vocabulário é uma maneira indireta de acesso à linguagem do indivíduo. A detecção precoce de atrasos de linguagem, verificada por meio da quantificação do vocabulário, permite a intervenção precoce por profissionais habilitados. Gatti (2004) mostra a existência de problemas educacionais que, para sua contextualização e compreensão, necessitam ser qualificados através de dados quantitativos. Mota e Castro (2007) e Giusti e Befi-Lopes (2008) denunciam a falta de instrumentos que possam ajudar o pesquisador e o profissional a estudarem e identificarem com mais eficácia os atrasos de linguagem.

Azanha (2004) afirma que as deficiências detectadas em relação à escola e ao alcance de seus objetivos precisam ser enfrentadas por um esforço permanente de investigação e busca. O objetivo do presente estudo, portanto, foi avaliar o desempenho de crianças pré-escolares quanto ao vocabulário receptivo antes do início do processo de alfabetização, procurando detectar possíveis atrasos de linguagem e verificar a interferência do gênero e de condições sociais no voca-

172 bulário das crianças.
Método

Estudo observacional transversal, aprovado pelo Comitê de Ética em pesquisa da Unifesp, sob o no 1042/03.

\section{Participantes}

De um universo de 330 alunos matriculados em uma escola municipal de educação infantil de São Paulo, participaram deste estudo, no ano letivo de 2004, 159 $(48,2 \%)$ alunos de quatro a sete anos de idade, sendo 84 $(53,0 \%)$ do gênero masculino e 75 (47,0\%) do feminino. Foram divididos em dois grupos de acordo com a escolaridade: o dos frequentadores do primeiro estágio - série inicial na educação infantil (56,0\%) e o dos frequentadores do terceiro estágio - última série da educação infantil (44,0\%). Com o ensino fundamental de nove anos, as crianças do terceiro estágio seriam consideradas alunas do primeiro ano.

\section{Instrumentos}

Foi utilizado para a avaliação o Teste de Vocabulário por Imagens Peabody (TVPI) (Capovilla et al., 1997), que avalia o desenvolvimento lexical no domínio receptivo de crianças entre dois anos e seis meses até dezoito anos de idade. O teste consiste de 130 pranchas com quatro desenhos cada uma, organizadas em grau crescente de dificuldade; o examinando aponta a figura que representa a palavra falada pelo examinador e, no final, cada examinando recebe um escore e uma classificação do desempenho de acordo com a faixa etária comparada à população de padronização. O instrumento permite uma avaliação objetiva, rápida e precisa do vocabulário receptivo auditivo em uma ampla variedade de áreas, incluindo pessoas, ações, qualidades, partes do corpo, tempo, natureza, lugares, objetos, animais, termos matemáticos, ferramentas e instrumentos.

\section{Procedimentos}

Inicialmente foi feito contato com a direção da escola para obter autorização para a realização da pesquisa. Em uma reunião de pais e mestres, foram expostos o objetivo do presente trabalho e o modo como ele seria realizado. Uma das pesquisadoras parti- 
cipou, durante duas semanas, das atividades escolares a fim de conhecer a dinâmica do estabelecimento e ser conhecida pelas crianças. Após autorização dos pais, as crianças que frequentavam o primeiro ou o terceiro estágio da educação infantil foram convidadas a acompanhar a pesquisadora a uma sala adequada na própria escola e a responderem ao instrumento. Foi permitida a presença da professora de classe ou de algum outro funcionário da escola a fim de dar segurança à criança e assegurar a ética do estudo. A pesquisadora permaneceu por quatro semanas na escola. A avaliação com cada criança demorou de 10 a 20 minutos para ser realizada e os dados foram anotados em um protocolo específico, que foi codificado, a fim de preservar o sigilo do estudo. Avaliaram-se pelo coeficiente de correlação de Pearson e pelo teste $t$ de Student as eventuais relações entre as variáveis. Após a análise dos dados, as pesquisadoras participaram do Horário de Trabalho Pedagógico Coletivo (HTPC) a fim de passar informações aos professores sobre o desenvolvimento da linguagem receptiva e mostrar os resultados do estudo, reafirmando o cumprimento dos princípios éticos que norteiam a pesquisa em psicologia.

\section{Resultados}

A média geral de idade das crianças avaliadas foi de 68 meses, com idade mínima de 47 e máxima de 95 meses, ocorrendo diferença estatisticamente significante entre as idades dos dois grupos $(p<0,0001)$. Não houve diferença estatisticamente significante na distribuição dos gêneros nos estágios (Tabela 1).

Tabela 1. Distribuição da amostra em relação ao sexo e idade, por escolaridade.

\begin{tabular}{|c|c|c|c|c|c|c|}
\hline \multirow{2}{*}{ Variáveis } & \multicolumn{2}{|c|}{$1^{\circ}$ estágio } & \multicolumn{2}{|c|}{$3^{\circ}$ estágio } & \multicolumn{2}{|c|}{ Total } \\
\hline & $n$ & $\%$ & $n$ & $\%$ & $n$ & $\%$ \\
\hline \multicolumn{7}{|l|}{ Sexo } \\
\hline Masculino & 41 & 46,1 & 34 & 48,6 & 75 & 47,2 \\
\hline Feminino & 48 & 53,9 & 36 & 51,4 & 84 & 53,8 \\
\hline \multicolumn{7}{|c|}{ Idade (anos) } \\
\hline 4 & 56 & 62,9 & 0 & 0,0 & 56 & 35,2 \\
\hline 5 & 33 & 37,1 & 0 & 0,0 & 33 & 27,7 \\
\hline 6 & 0 & 0,0 & 49 & 70,0 & 49 & 30,8 \\
\hline 7 & 0 & 0,0 & 21 & 30,0 & 21 & 13,2 \\
\hline Total & 89 & 56,0 & 70 & 44,0 & 159 & 100,0 \\
\hline
\end{tabular}

Tabela 2. Desempenho no Teste de Vocabulário por imagens (TVIP) em relação ao sexo, idade, escolaridade, ocupação materna e escolaridade materna.

\begin{tabular}{|c|c|c|c|c|c|c|c|c|}
\hline \multirow{2}{*}{ Variáveis } & \multicolumn{2}{|c|}{ Abaixo da média } & \multicolumn{2}{|c|}{ Média } & \multicolumn{2}{|c|}{ Acima da média } & \multicolumn{2}{|c|}{ Total } \\
\hline & $n$ & $\%$ & $n$ & $\%$ & $\mathrm{n}$ & $\%$ & $n$ & $\%$ \\
\hline \multicolumn{9}{|l|}{ Sexo } \\
\hline Masculino & 47 & 62,7 & 25 & 33,3 & 3 & 4,0 & 75 & 47,2 \\
\hline Feminino & 50 & 59,5 & 31 & 36,9 & 3 & 3,6 & 84 & 53,8 \\
\hline \multicolumn{9}{|l|}{ Idade (anos) } \\
\hline 4 & 24 & 42,9 & 27 & 48,2 & 5 & 8,9 & 56 & 35,2 \\
\hline 5 & 20 & 60,5 & 12 & 36,4 & 1 & 3,0 & 33 & 27,7 \\
\hline 6 & 36 & 73,5 & 13 & 26,5 & 0 & 0,0 & 49 & 30,8 \\
\hline 7 & 17 & 81,0 & & 19,0 & 0 & 0,0 & 21 & 13,2 \\
\hline \multicolumn{9}{|l|}{ Escolaridade } \\
\hline $1^{\circ}$ estágio & 44 & 49,4 & 39 & 43,8 & 6 & 6,7 & 89 & 56,0 \\
\hline $3^{\circ}$ estágio & 53 & 75,7 & 17 & 24,3 & 0 & 0,0 & 70 & 44,0 \\
\hline \multicolumn{9}{|c|}{ Ocupação materna } \\
\hline Empregada & 38 & 59,4 & 23 & 35,9 & 3 & 4,7 & 64 & 40,3 \\
\hline Desempregada & 27 & 60,0 & 16 & 35,6 & 2 & 4,4 & 45 & 28,3 \\
\hline Sem dados & 32 & 64,0 & 17 & 34,0 & 1 & 2,0 & 50 & 31,4 \\
\hline \multicolumn{9}{|c|}{ Escolaridade materna } \\
\hline Até 5 anos & 15 & 60,0 & 10 & 40,0 & 0 & 0,0 & 25 & 15,7 \\
\hline Maior de 5 anos & 47 & 58,7 & 28 & 35,0 & 5 & 6,3 & 80 & 50,2 \\
\hline Sem dados & 35 & 64,8 & 18 & 33,3 & 1 & 1,9 & 54 & 34,0 \\
\hline Total & 97 & 61,0 & 56 & 35,2 & 6 & 3,8 & 159 & 100,0 \\
\hline
\end{tabular}


A escolaridade média das mães dos participantes era de sete anos e meio, sendo a maior escolaridade de 13 anos de frequência escolar, e a menor de um ano de estudo. Não houve diferença estatisticamente significante em relação à escolaridade materna, ao gênero das crianças ou ao estágio escolar. O mesmo ocorreu com o fato de a mãe trabalhar ou não fora de casa. A mãe mais nova tinha 20 anos (quinze anos de diferença em relação ao filho) e a mais velha, 50 (46 anos de diferença em relação ao seu filho).

Houve um desempenho inferior ao esperado para a idade no TVIP em 61,0\% das crianças da pré-escola. No primeiro estágio, 49,4\% dos alunos tiveram um desempenho inferior; e no terceiro, 75,7\%.

Esse teste apresenta uma pontuação máxima de acerto em 125 pontos. As crianças do terceiro estágio tiveram um número de palavras corretas significantemente maior ( $p<0,001)$ - média de 59 palavras - que as do primeiro estágio - média de 39 palavras. Apesar de terem acertado mais palavras, houve uma associação negativa entre a escolaridade e o desempenho no teste ( $p=0,001)$ : os alunos do terceiro estágio saíram-se pior que os do primeiro estágio. Seis crianças que frequentavam o primeiro estágio foram classificadas com desempenho superior (Tabela 2).

A maioria das crianças com quatro anos de idade obteve pontuação na média e a maioria das crianças de cinco e sete anos obtiveram pontuação baixa. Não houve diferença estatística significante entre as médias de acertos por gênero $(p=0,94)$ - tanto meninos quanto meninas acertaram em média 47 palavras - nem quanto ao desempenho no teste.

O grau de escolaridade da mãe não interferiu no número de acertos ou desempenho do teste dos filhos $(p=0,42)$, assim como o fato de ela trabalhar ou não fora de casa $(p=0,99)$.

\section{Discussão}

A avaliação do vocabulário é um indicador do desenvolvimento cognitivo da criança e pode servir para nortear políticas educacionais para a educação infantil, inclusive prevenindo problemas escolares futuros. Concordamos com Gatti (2004) de que são necessários dados quantitativos para compreender melhor os proble-

174 mas educacionais brasileiros.
Este estudo confirmou que, com o aumento da idade, aumenta-se também o vocabulário, que é um fator do próprio desenvolvimento (Atance \& Jackson, 2009; Macedo et al., 2006; Rhoades et al., 2009). Entretanto, o fato de as crianças mais velhas terem tido um desempenho pior que as mais novas é um indicador de que quantidade não significa qualidade. Para haver uma boa comunicação entre as pessoas, não basta ter mais palavras, mas que o vocabulário acompanhe, de forma harmônica, as exigências da cultura em que a pessoa está inserida.

Sem esquecer os limites impostos por um estudo transversal como este, parece que o baixo desempenho dos alunos do último estágio da pré-escola denuncia a falta de habilidades que deveriam ter sido desenvolvidas ao longo desse nível educacional. A criança ingressa no sistema educacional com um vocabulário pobre, e a escola não dá conta de diminuir a defasagem existente. De acordo com Belintane (2000), a criança necessita de um vocabulário mais amplo para transitar bem em diversos ambientes. Acreditamos que, com essa defasagem no vocabulário, torna-se difícil a criança transitar adequadamente no ambiente escolar, e, consequentemente, ela poderá apresentar problemas de aprendizagem durante o ensino fundamental. Um trabalho voltado para o aumento do léxico na educação infantil é fundamental para facilitar a alfabetização.

Comparando esses achados aos do estudo realizado por Basílio et al. (2005), verificou-se que as crianças do Embu apresentaram desempenho superior. Em ambos, as crianças apresentaram um alto índice de problemas com relação à linguagem receptiva, medida pelo TVIP. Esses resultados nos levam a refletir se a amostra padronizada do TVIP é muito diferente das crianças deste estudo e, portanto, se seria inviável uma comparação, ou se o local onde os estudos estão sendo realizados são ambientes de risco para o desenvolvimento. Há uma forte associação positiva entre qualidade da estimulação ambiental e desempenho cognitivo infantil.

Os estudos vêm demonstrando que crianças advindas de meios socioculturais baixos apresentam um risco maior de terem problemas de desenvolvimento, inclusive de desenvolvimento da linguagem. Essas crianças podem ser beneficiadas não com uma outra padronização no TVIP, mas com intervenções na escola de educação infantil que visem ao desen- 
volvimento integral e procurem minimizar as diferenças existentes entre as crianças advindas de meios socioculturais mais privilegiados.

É importante, portanto, um trabalho de intervenção já na pré-escola a fim de diminuir a distância entre o vocabulário apresentado pelas crianças e o que será exigido no ensino fundamental, pois, com a progressão escolar, aumentam-se as dificuldades escolares, favorecendo um pior desempenho escolar por parte das crianças que já ingressam apresentando uma história de déficits.

Acreditamos ser importante evitar que essas dificuldades se agravem, e procurar uma intervenção o mais precocemente possível. Concordamos com Azanha (2004) de que é necessário uma melhor formação do professor de educação infantil, incluindo estratégias para trabalhar com crianças com problemas de desenvolvimento na educação regular.

Adquirir palavras não é somente falar, mas compreender a palavra dita e procurá-la na memória. É também utilizar as habilidades metalinguísticas para concluir algo a respeito dos vocábulos ouvidos. Não se pode ser passivo no tocante à linguagem. Há sempre a necessidade de o indivíduo utilizar sua capacidade cognitiva para compreender e interferir no mundo. Sem um repertório amplo de palavras, fica difícil para a criança criar novas categorias conceituais (Bee, 2003). Com poucas categorias, a busca de significado e compreensão torna-se mais lenta, dificultando a aquisição de regras para a formação de palavras ou para acompanhar os assuntos discutidos em contextos formais (Bee, 2003; Berk, 2001).

Observou-se, neste estudo, a necessidade de investimento em intervenções que alcancem as crianças pequenas antes que elas fracassem no sistema educacional. É necessário preparar o professor para enfrentar este desafio: favorecer, através da educação, a inserção do indivíduo à sociedade organizada.

\section{Considerações Finais}

A maioria das crianças que participaram deste estudo teve seu vocabulário receptivo classificado como abaixo da média, especialmente as mais velhas.

Embora este trabalho tenha dado ênfase às crianças que tiveram um desempenho abaixo do espe- rado para a idade, não se deve negligenciar o estudo das crianças que apresentaram um desempenho superior, pois elas também se beneficiariam com uma educação de qualidade.

\section{Referências}

American Psychiatric Association. (2002). Manual diagnóstico e estatístico de transtornos mentais - DSM-IVTR (4a ed.). Porto Alegre: Artmed.

Atance, C. M., \& Jackson, L. K. (2009). The development and coherence of future-oriented bahaviors during the preschool years. Journal of Exprerimental Child Psychology, 102, 379-391.

Azanha, J. M. P. (2004). Uma reflexão sobre a formação do professor da escola básica. Educação e Pesquisa, 30 (2), 369-378.

Basílio, C. S., Puccini, R. F., Silva, E. M. K., \& Pedromônico, M. R. M. (2005). Living conditions and receptive vocabulary of children aged two to five years. Revista de Saúde Pública, 39 (5), 725-730.

Bee, H. (2003). A criança em desenvolviment (9a ed.). Porto Alegre: Artmed.

Belintane, C. (2000). Linguagem oral na escola em tempo de redes. Educação e Pesquisa, 26 (1), 53-65.

Benedict, H. (1979). Early lexical development: comprehension and production. Journal of Child Language, 6 (2), 183-200.

Berk, L. E. (2001). Desarrollo del niño y del adolescente (4a ed.). Madrid: Prentice-Hall Iberia.

Capovilla, F. C., \& Capovilla, A. G. S. (1997). Desenvolvimento linguístico na criança dos dois aos seis anos: tradução e estandardização do Peabody Picture Vocabulary Test de Dunn \& Dunn, e da Language Development Survey, de Rescorla. Ciência Cognitiva: Teoria, Pesquisa e Aplicação, 1 (1), 353-380.

Capovilla, F. C., Capovilla, A. G. S., Nunes, L., Araujo, I., Nunes, D., Nogueira, D., et al. (1997). Versão brasileira do teste de vocabulário por imagens Peabody. Revista Distúrbios da Comunicação, 8 (2), 151-162.

Cardoso, R. M., Pedromônico, M. R. M., Silva, E. M. K., \& Puccini, R. F. (2003). Conhecimento de mães e auxiliares de desenvolvimento infantil referente ao desenvolvimento da linguagem de crianças de zero a vinte e quatro meses. Revista Brasileira de Crescimento e Desenvolvimento Humano, 13 (2), 85-94.

Chapman, R. S. (1996). Processos e distúrbios na aquisição da linguagem. Porto Alegre: Artes Médicas.

Dias, T. L., Enumo, S. R. F., \& Turini, F. A. (2006). Avaliação do desempenho acadêmico de alunos do ensino fundamental em Vitória, Espírito Santo. Estudos de Psicologia (Campinas), 23 (4), 381-390.

Duncan, J. D., Brooks-Gunn, J., \& Klebanov, P. K. (1994). Economic deprivation and early childhood development. Child Development, 65 (2), 296-318. 
Feldman, H. M., Campbell, T. F., Kurs-Lasky, M., \& Rockette (2005). Concurrent and predictive validity of parent reports of child language at ages 2 and 3 years. Child Development, 76 (4), 856-868.

Fitch, W. T. (2007). Linguistics: an invisible hand. Nature, 449 (11), 665-667.

Gatti, B. A. (2004). Estudos quantitativos em educação. Educação e Pesquisa, 30 (1), 11-30.

Giusti, E., \& Befi-Lopes, D. M. (2008). Tradução e adaptação transcultural de instrumentos estrangeiros para o português brasileiro (PB). Pró-Fono Revista de Atualização Científica, 20 (3), 207-210.

Hage, S. R. V., Joaquim, R. S. S., Carvalho, K. G., Padovani, C. R., \& Guerreiro, M. M. (2004). Diagnóstico de crianças com alterações específicas de linguagem por meio de escala de desenvolvimento. Arquivos de Neuropsiquiatria, 62 (3-A), 649-653.

Johnson, K. N., Karrass, J., Conture, E. G., \& Walden, T. (2009). Influence of stuttering variation on talker group classification in preschool children: preliminary findings. Journal of Communication Disorders, 42, 195-210.

Li, P.,Zhao, X., \&Whinney, B.M. (2007). Dynamic self-organization and early lexical development in children. Cognive Science, 31 (4), 581-612.

Luque, A., \& Vila, I. (2004). Desenvolvimento da linguagem. In C. J. Palácios \& A. Marchesi (2004), Desenvolvimento psicológico e educação: psicologia evolutiva (2a ed., Vol. 1). Porto Alegre: Artmed.

Macedo, E. C., Capovilla, F. C. Duduchi, M., D’Antino, M. E. F., \& Firmo, L. S. (2006). Avaliando linguagem receptiva via teste de vocabulário por imagens Peabody: versão tradicional versus computadorizada. Psicologia: Teoria e Prática, 8 (2), 40-50.

Mota, M. M. E. P., \& Castro, N. R. (2007). Alfabetização e consciência metalinguística: um estudo com adultos não alfabetizados. Estudos de Psicologia (Campinas), 24 (2), 169-179.
Nogueira, S., Fernández, B., Porfírio, H., \& Borges L. (2000). A criança com atraso da linguagem. Saúde Infantile, 22 (1), $5-16$.

Olds, D. L., Kitzman, H., Cole, R., Robinson, J. Sidora, K. Luckey, D., et al. (2004). Effects of Nurse home-Visiting on maternal life course and child development: age 6 follow-up results of a randomized trial. Pediatrics, 114 (6), 1550-1559.

Perissinoto, J., Schoen-Ferreira, T. H., \& Marteleto, M. R. F. (2007). Incidência de problemas de fala ou articulação em crianças/adolescentes do Município de São Paulo. Anais do $15^{\circ}$ Congresso Brasileiro de Fonoaudiologia, 2007, Gramado-RS. Suplemento Especial da Revista da Sociedade Brasileira de Fonoaudiologia.

Rhoades, B. L., Greenberg, M. T., \& Domitrovich, C. E. (2009). The contribution of inhibitory control to preschoolers'social-emotional competence. Journal of Applied Developmental Psychology, 30 (3), 310-320.

Romski, M. A., \& Sevcik, R. A. (2005). Augmentative Communication and early intervention: myths and realities. Infants \& Young Children, 18 (3), 174-185.

Schirmer, C. R., Fontoura, D. R., \& Nunes, M. L. (2004) Distúrbios da aquisição da linguagem e da aprendizagem. Jornal de Pediatria, 80 (2 Supl), S95-S103.

Smeekens, S., Riksen-Walraven, J. M., \& van Bakel, H. J. A. (2008). Profiles of competence and adaptation in preschoolers as related to the quality of prent-child interaction. Journal of Research in Personality, 42 (6), 14901499.

Sternberg, R. J., \& Grigorenko, E. L. (2004). Why we need to explore development in its cultural context. Journal of Developmental Psychology, 50 (3), 369-386.

Sternberg, R. J., \& Grigorenko, E. L. (2006). Cultural Intelligence and successful intelligence. Group \& Organization Management, 31 (1), 27-39.

Recebido em: 14/12/2007

Versão final reapresentada em: 29/6/2009

Aprovado em: 23/9/2009 